\title{
Parents and Early Childhood Professionals Want a Website on Torticollis
}

ISSN: 2576-9200

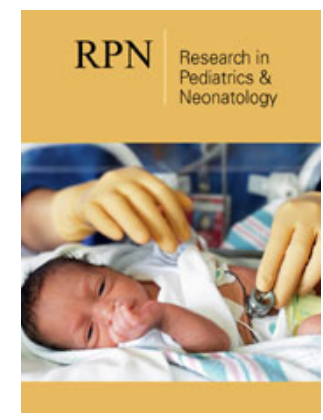

*Corresponding author: Brenda HusseyGardner, Department of Pediatrics, University of Maryland School of Medicine, 110 South Paca Street-8th Floor, Baltimore, MD, USA 21201

Submission: 眥 October 11, 2019

Published: N November 07, 2019

Volume 3 - Issue 5

How to cite this article: Vu KTT, Seo YJ, Hussey-Gardner B. Parents and Early Childhood Professional Want a Website on Torticollis. Research Article, Res Pediatr Neonatol. 3(5).RPN.000575.2019. DOI: 10.31031/RPN.2019.03.000575

Copyright $($ C Brenda Hussey-Gardner This article is distributed under the terms of the Creative Commons Attribution 4.0 International License, which permits unrestricted use and redistribution provided that the original author and source are credited.

\author{
Kathy T. T. Vu ${ }^{1}$, You Jung Seo ${ }^{1}$ and Brenda Hussey-Gardner ${ }^{2 *}$ \\ ${ }^{1}$ University of Maryland, Baltimore County \\ ${ }^{2}$ University of Maryland School of Medicine
}

\begin{abstract}
Purpose: There is a lack of research on the need for and desirability of a website on torticollis for parents. Thus, this study determined the desirability of a website with information for parents of young children on torticollis.

Methods: Parents $(n=226)$ and professionals who work with children ages birth-to-5 years $(n=68)$ completed an online survey.

Results: Most parents (76\%) and professionals (76\%) reported knowing nothing or very little about torticollis. Ninety-five percent of parents thought that a website could help parents learn more about torticollis. However, parents and professionals thought that a website tailored towards parents was needed and that current available resources were not helpful or needed improvement. Both parents and professionals identified key information that the website should include.
\end{abstract}

Conclusion: Both parents and professionals expressed a desire for a website on torticollis that is tailored towards parents.

Keywords: Torticollis; Website; Parenting; Physical therapy; Pediatrics

\section{Introduction}

Congenital muscular torticollis is a condition in which the sternocleidomastoid muscle (SCM), a muscle in the neck, is shortened and as a result, the head is tilted toward one side and rotated away from the shortened SCM [1]. This muscle may have been shortened at birth due to intrauterine crowding or shortly after birth. In contrast, acquired torticollis develops when there is a strong positional preference of the head due to a wide range of underlying issues, such as infection and trauma, and often occurs after the first 2 months of life [1,2]. However, both forms of torticollis are characterized by unnatural head and neck position and may lead to plagiocephaly (flattened skull), imbalance, hip dysplasia, vision and hearing problems, and scoliosis $[2,3]$. Early and immediate treatment of both forms of torticollis, specifically before 3 months of age, is suggested and encouraged [4].

Due to the similarities between the two forms of torticollis, we will use the term torticollis to refer to both. The incidence of torticollis ranged between $0.3 \%$ to $2 \%$ in older studies [5] but has increased to $16 \%$ in normal newborn populations in the recent years [6,7]. The Academy of Pediatric Physical Therapy recently released clinical practice guidelines for the diagnosis of torticollis [8]. Although the clinical practice guidelines were designed to help parents and caregivers in addition to healthcare providers, this resource seems to be mainly directed towards healthcare providers (e.g., due to technical jargon) and may be overwhelming to parents. As such, parents may not have easy access to jargon-free high-quality information and resources that can inform them about torticollis and available treatments for this condition. Therefore, in this study, we aimed to provide preliminary evaluations of the desirability of a website for parents on torticollis.

\section{Role of websites in increasing parental knowledge}

Websites provide easily accessible information to the general population. Research examining the usefulness of websites on other child-related outcomes, such as diabetes and obesity, revealed that the websites were viewed as valuable by parents and increased parental self-efficacy $[9,10]$. Furthermore, website-based interventions with parents on children's 
oral care and obesity increased parental knowledge, which may promote treatment compliance and help-seeking behaviors $[9,11]$. Importantly, it is necessary for parents to be able to first identify the symptoms of torticollis in their infants in order to seek professional help and start treatment of the condition [12]. A website on torticollis can provide easily accessible information to all parents. This open access to information on torticollis may increase parental knowledge about torticollis and parental self-efficacy, which may promote the successful treatment of children's torticollis.

Parents may feel more comfortable when they have more information on torticollis including therapeutic strategies and expected outcomes, prior to meeting with professionals [1]. Treatment of torticollis in infants includes repeated stretching exercises with trained physical and occupational therapists [13]. Because infants may be more comfortable if parents conduct stretching exercises in familiar settings, and to supplement therapy sessions, many professional providers encourage parents to use stretching exercises with their infants at home [14]. Parents of children with other physical disabilities perceive at-home exercises with their children as more successful when they have greater positive experiences with their physical therapists [15]. Parents' positive feelings about these at-home exercises, as well as feelings of efficacy, may be bolstered if they also have additional knowledge on their child's condition. Previous early intervention research found that parents highlighted the importance of having access to various intervention services aside from therapy and the need for greater clarity about what to expect from services (e.g., intensity of therapy, length of services) [16]. However, it is unknown whether such needs are relevant to parents of children with torticollis.

\section{Current websites on torticollis}

A review on Google and research databases (i.e., PsycINFO and MEDLINE) revealed that current websites on torticollis [17-21] provide limited information and do not offer and/or refer parents to additional resources (e.g., local early intervention programs) that may facilitate their children's treatment. In addition, these websites utilize complex medical terminology that may not be easily understood by and may be overwhelming for the general parent population. Thus, parents may not have access to high-quality information and resources that can inform them about torticollis and available treatments for this condition. Studies show that webbased information is more effective in conveying messages if the information provided is tailored toward the population of interest (i.e., parents) $[11,16]$.

Although past research suggests that websites may be a useful tool in delivering information to parents $[22,23]$ there is a lack of research on the need for and desirability of a website on torticollis for parents. Moreover, there is a dearth of information regarding aspects of such a website desired by parents and early childhood professionals. An evidence-based website on torticollis that is tailored toward parents may be a particularly informative and effective tool in increasing parental knowledge and efficacy. Thus, evaluating the desirability of a website on torticollis and important website features on torticollis will provide preliminary empirical evidence for the need to improve current or develop new websites on torticollis.

\section{The present study}

The purpose of this study was to assess desirability of a website on torticollis for parents of young children and identify important features or information to be included on a website on torticollis. Specifically, the first aim of this study was to provide preliminary assessments of parents' and early childhood professionals' desire for a website with information for parents of infants and young children on torticollis. The second aim of this study was to identify and determine what information parents and professionals believe would be important to include on the website.

\section{Methods}

\section{Procedures}

Participants were recruited through letters and flyers sent to directors of local early intervention programs, Early Head Start and Head Start programs, childcare centers, family support centers, and pediatric practices to be given to parents and early childhood professionals. Social media (i.e., Facebook, Reddit) postings were also used to recruit parents and early childhood professionals. To be eligible for the study, participants had to be a legal guardian of a child 5 years of age or younger or a professional who works with children 5 years of age or younger. Participants must also be at least 18 years old and be able to read and write in English to participate. This study was reviewed by Institutional Review Board (IRB) and determined to be exempt. All participants filled out informed consent forms and completed an online SurveyMonkey survey (January 2018 to May 2018).

\section{Participants}

Participants included 226 parents $\left(M_{\text {age }}=34.34\right.$ years, $\left.S D=4.38\right)$ and 68 professionals who work with children ages birth-to-5 years $\left(M_{\text {age }}=36.65\right.$ years, $\left.S D=11.93\right)$. Parents were comprised of mothers (96\%), fathers (3\%) and grandmothers (1\%). Most parents selfidentified as White or Caucasian (81\%) whereas 11\% identified as Asian, $3 \%$ as Hispanic or Latinx, $1 \%$ as Black, $1 \%$ as Native American or Alaskan Native, $1 \%$ as Native Hawaiian or other Pacific Islander, $1 \%$ as $\mathrm{Bi} / \mathrm{Multiracial}$, and $1 \%$ preferred not to answer. Most parents $(88 \%)$ were from middle-to-upper class families $(\geq \$ 50,000$ yearly income). Professionals were comprised of teachers (e.g., Early Head Start; 37\%), therapists (e.g., occupational, speech and physical; $26 \%)$, childcare providers (18\%), physicians and nurses (13\%), service coordinator/case managers and social workers (10\%), psychologists $(6 \%)$, and doulas (2\%). Most professionals selfidentified as female (92\%), and White or Caucasian (83\%) whereas $5 \%$ as Asian, 5\% Hispanic or Latinx, 3\% Black, 1\% Bi/Multiracial, and $3 \%$ preferred not to answer.

\section{Measures}

There were two, almost identical, online SurveyMonkey surveys included in the present study; one for parents (Appendix A) and one for professionals (Appendix B). The questions were developed by individuals with expertise in child development and evaluated 
for face validity. The parent questionnaire contained 17 questions asking parents to report on their knowledge regarding torticollis, where they turn to first for help when their child is sick or when they have questions about their child's development, whether they think a website would help parents learn about torticollis, and current available resources for parents of children with torticollis. In addition, parents rated their endorsement of 5 different types of information (Table 1) on a 5-point Likert-type scale (1=not important to 5 =important, Table 1) and ranked 5 website features (Table 2). The professional survey contained 15 items. Aside from basic demographic information, the only differences between this survey and the parent survey was professionals' report of:

1. Whether they've worked with a child with torticollis before;

2. The importance of providing information regarding torticollis to parents as a professional; and

3. Additional ranking of other resources and organizations that may provide additional assistance to families of children with torticollis (Table 3).

Table 1: Parents' and professionals' endorsement of the importance of specific information.

\begin{tabular}{|c|c|c|}
\hline The Importance of Providing Information on: & Parents & Professionals \\
\hline 1. Non-normal development pertaining to torticollis & $91 \%$ & $94 \%$ \\
\hline 2. What to expect when professionals assess children for torticollis & $95 \%$ & $100 \%$ \\
\hline 3. What steps to take if their child has torticollis & $98 \%$ & $100 \%$ \\
\hline 4. Suggestions and activities to help parents improve their child's torticollis utilizing photographs & $97 \%$ & $92 \%$ \\
\hline 5. Available local program and family resources (e.g., early intervention) & $93 \%$ & $97 \%$ \\
\hline
\end{tabular}

Note: Percentages reflect the percent of parents or professions who thought it was important that the website provided the specific website feature or information.

Table 2: Parents' and professionals' rankings of additionally desired torticollis website features.

\begin{tabular}{|c|c|c|}
\hline Website Features & Parents & Professionals \\
\hline 1. List of exercises to do with child & $\begin{array}{c}1 \\
(31 \%)\end{array}$ & $\begin{array}{c}3 \\
(17 \%)\end{array}$ \\
\hline 2. List of what to expect at assessment with pediatrician & $\begin{array}{c}2 \\
(26 \%)\end{array}$ & $\begin{array}{c}2 \\
(29 \%)\end{array}$ \\
\hline 3. Resources list (e.g., systems of early intervention) & $\begin{array}{c}3 \\
(17 \%)\end{array}$ & $\begin{array}{c}1 \\
(32 \%)\end{array}$ \\
\hline 4. List of what to expect at appointments with pediatricians and physical therapist & $\begin{array}{c}4 \\
(16 \%)\end{array}$ & $\begin{array}{c}4 \\
(15 \%)\end{array}$ \\
\hline 5. List of what to expect at assessment with physical therapist & $\begin{array}{c}5 \\
(6 \%)\end{array}$ & $\begin{array}{c}5 \\
(6 \%)\end{array}$ \\
\hline
\end{tabular}

Note: Percentages reflect the percent of parents or professions who ranked the specific website feature as rank 1.

\section{Results}

\section{The importance of a website on torticollis}

Parents: The majority of parents (76\%) reported knowing nothing or very little about torticollis. Many parents (44\%) reported turning to professionals, such as their pediatrician, for help when they have questions about their child's development but 34\% reported turning to the Internet when they have questions. In addition, $96 \%$ of parents thought that a website could help parents learn more about torticollis and $88 \%$ said they would use such a website to identify if their child could potentially have torticollis. A limited number of parents $(30 \%)$ reported having used a website on torticollis but said that the website was only somewhat helpful or not helpful at all. Most parents (93\%) thought it was important that parents have easy and free access to information about torticollis.

Professionals: Similar to parents, $76 \%$ of professionals reported knowing nothing or very little about torticollis, yet almost half (47\%) reported having previously worked with a child with 
torticollis. Moreover, $96 \%$ of professionals thought that a website could help parents learn more about torticollis and 19\% thought that the current resources (e.g., websites) available for parents of children with torticollis were not helpful or needed improvement. Most professionals (91\%) thought it was important that parents have easy and free access to information about torticollis and 74\% thought it was important that they, as a professional, should inform all parents about torticollis.

\section{Important website aspects}

The specific information parents and professionals viewed as important to include on a website was calculated by adding up the percentage of parents and professionals who endorsed options 4 (somewhat important) and 5 (important) and are reported in Table 1. Both parents and professionals thought it was important that the website provide information on:

1. Non-normal development pertaining to torticollis;

2. What to expect when professionals assess children for torticollis;

3. What steps to take if their child has torticollis;
4. Suggestions and activities to help parents improve their child's torticollis utilizing photographs; and

5. Available local program and family resources.

Parents most desired information on what steps to take if their child has torticollis and suggestions and activities, they can engage in to help improve their children's torticollis through photographs. In contrast, professionals most desired information for parents on what steps to take if their child has torticollis and on what to expect when professionals assess children for torticollis. Parents and professionals also ranked other desired website features. Rankings of other desired website features were calculated by adding up the percentages of parents and professionals who ranked each feature as 1 (most desired) and are reported in Table 2. The top two parent requested website features were a list of exercises to do with their child and what to expect at an assessment with the pediatrician. In contrast, the top two professional requested features included a resource list and a list of exercises to do with the child. In addition, professionals ranked the types of resources to include in the list; early intervention ranked as first (Table 3 for the top 5 requested resources).

Table 3: Professionals' top 5 ranking of important organizations and resources.

\begin{tabular}{|c|c|}
\hline Rank & Resource \\
\hline $\begin{array}{c}1 \\
(66 \%)\end{array}$ & $\begin{array}{l}\text { Early Intervention: A family centered system of services for young children with developmental delays and disabilities and } \\
\text { their families }\end{array}$ \\
\hline $\begin{array}{c}2 \\
(11 \%)\end{array}$ & Pediatrician \\
\hline $\begin{array}{c}3 \\
(7 \%)\end{array}$ & $\begin{array}{l}\text { Home Visiting Program: Supports for pregnant women and families, and helps parents of children birth to age } 5 \text { tap re- } \\
\text { sources and develop skills needed to raise children who are physically, socially and emotionally healthy and ready to learn }\end{array}$ \\
\hline $\begin{array}{c}4 \\
(6 \%)\end{array}$ & Early Head Start/Head Start: Educational program for children from families with low income \\
\hline $\begin{array}{c}5 \\
(6 \%)\end{array}$ & $\begin{array}{l}\text { Supplemental Security Income: Program designed to help families who have children with disabilities and have little or no } \\
\text { income meet their basic needs for food, clothing, and shelter }\end{array}$ \\
\hline
\end{tabular}

Note: Percentages reflect the percent of professions who ranked the specific resource as rank 1.

\section{Discussion}

The present study assessed parents' and professionals' desire for a website with information for parents of young children on torticollis and identified information parents and professionals believed was important to include on such a website. We found that an overwhelming majority of parents had little-to-no knowledge about torticollis despite the increasing rates of torticollis [6,7]. More surprising, most early childhood professionals in our sample had little-to-no knowledge about torticollis even though almost half reported having worked with a child with torticollis. This finding suggests that more work is needed to inform both parents and early childhood professionals about torticollis and the importance of early identification and treatment of this serious condition [4]. In addition, both groups emphasized the importance of free and easily accessible information about torticollis for parents, which can be provided through a website [11,22,23]. Collectively, our findings indicated that parents and professionals believed that a website on torticollis for parents is needed.

Many parents reported interest in using a website to identify if their child could potentially have torticollis but both parents and professionals thought that the current available resources, such as websites, were not helpful or needed improvement. Specifically, both parents and professionals felt that it was important that the website featured information on non-normal development pertaining to torticollis, expectations on what professionals do when assessing their child, what steps to take after their child 
has been diagnosed, at-home exercises that may increase the effectiveness of treatment, and available local program and family resources that parents can access for additional information and help $[15,16]$. In addition, parents and professionals highly ranked (i.e., ranks 1,2 and 3) at-home exercises with their child, a list of clear expectations during assessment of torticollis, and resources lists as important website features [16]. However, current websites on torticollis do not readily provide these resources to parents. A list of what to expect at appointments and at assessment with the physical therapist ranked 4 and 5 respectively. Parents' desire for these website features and resources support prior research findings highlighting the importance of parental role, knowledge and comfort in the therapeutic treatment of children's physical disabilities $[15,16]$. In addition, having easy access to additional resources may help parents conduct at-home exercises with their children to supplement therapy/treatment [14-16].

Professionals also emphasized the importance of providing several resources for parents on the website. Two-thirds of professionals felt that the website needed to provide parents with information on early intervention programs that provide services to young children with developmental delays and disabilities and their families. Other important resources that professionals emphasized should be provided by the website were pediatricians, home visiting programs, Early Head Start or Head Start programs, and Supplemental Security Income program. These resources may be essential in promoting children's successful treatment of torticollis and provide additional relief for parents and families $[9,11]$. Furthermore, professionals can use these website features and resources to supplement treatment and foster parents' positive communication and interactions with professionals, which has been found to promote greater adherence to treatment and exercise programs $[4,15,16]$. Unfortunately, current websites on torticollis do not provide key information and features captured by this study in a manner that is easily understood and accessible by parents. In addition to the aforementioned information and resources, the Academy of Pediatric Physical Therapy's clinical practice guidelines for the diagnosis of torticollis should also be included on the website as it provides key information to parents and professionals regarding the recommended state of diagnosis and treatment for physical therapy [8]. An example layout for the potential website on torticollis was included in Figure 1.
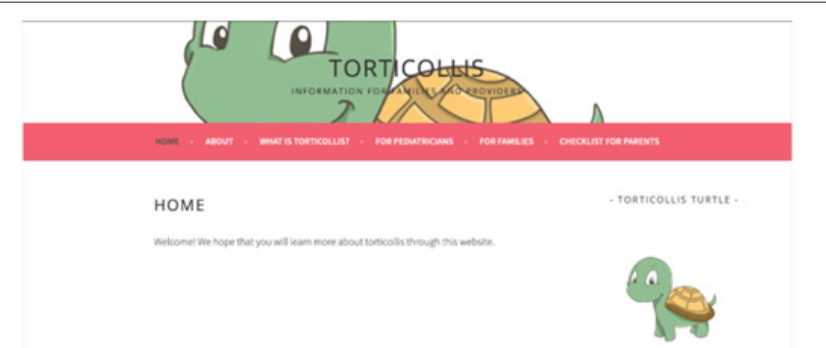

Figure 1: An example layout of the potential website on torticollis.

\section{Limitation and future directions}

Several limitations of this study should be noted. First, this study utilized only self-report questionnaires and the study findings were preliminary and descriptive. Interviews and focus groups with parents and professionals may provide more in-depth understanding of parents' needs. Furthermore, we are not able to make conclusions on the effectiveness of such a website due to the nature of our study. Future studies examining the effectiveness of a website on torticollis in increasing parental knowledge on torticollis is needed. Second, our sample was not very diverse in several aspects. Specifically, our study had only a few medical providers (13\%) who responded to our survey. Future studies should include a larger sample of medical providers who may be more familiar with torticollis and able to identify unique website features that may be important from a medical perspective.

Moreover, our parent sample was predominately comprised of White mothers and our professional sample was predominately White females. Underserved communities and minority populations tend to under-utilize available resources such as early intervention programs, due to lack of knowledge about children's developmental delays or lack of access to these resources. For instance, Asian immigrants are less likely to seek help from doctors due to barriers, such as language, immigrant status and health insurance [24]. Future studies should include more diverse samples to have a more comprehensive and representative understanding of parents' and professionals' needs and concerns.

\section{Conclusion}

Despite these limitations, the present study highlighted the importance of a website on torticollis that is tailored towards parents and provided preliminary evidence on the need for such a website. Importantly, both parents and professionals expressed a desire for a website on torticollis for parents. Moreover, our findings can inform the future development of a website for parents on torticollis and other developmental delays that promote parental knowledge and child development. Knowledge gained from this study will help guide the development of a parent website on torticollis in a manner that may best meet the needs of parents and professionals. When developing the website, physical therapists, the Academy of Pediatric Physical Therapy's clinical practice guidelines for the diagnosis of torticollis [8], and parents should be consulted to ensure that the website contains appropriate information for parents and professionals.

Moreover, the website should include information on nonnormal development pertaining to torticollis, what parents should expect when their child is assessed for torticollis, what parents should do if their child is diagnosed with torticollis, suggestions and activities parents can do at home with their child to improve their child's torticollis, and available local resources and supports. Moreover, the website should include lists of information pertaining to torticollis (e.g., exercises, resources, and expectations working with professionals) to help parents organize and prepare for assessments, appointments, and treatment. The website should be regularly updated following empirical studies on torticollis and 
clinical guidelines provided by the Academy of Pediatric Physical Therapy.

\section{Appendix A}

1. How much do you know about torticollis?

a. Nothing

b. A little

c. A lot

Torticollis is a condition in which the sternocleidomastoid muscle (SCM), a muscle in the neck, is shortened. As a result, the head is tilted toward one side and rotated away from the shortened SCM. This muscle may have been shortened at birth (due to intrauterine crowding) or developed shortly after birth. Early treatment of torticollis is suggested and encouraged. Some signs of trauma on this muscle include bruising and swelling. Torticollis may lead to plagiocephaly (flattened skull), imbalance, hip dysplasia, vision and hearing problems, and scoliosis.

2. When your child is sick or when you have questions about your child's development, where do you turn to first for help?

a. Parents or grandparents

b. Other family members

c. Friends

d. Other professionals (Please specify):

3. Do you think a website could help parents learn about torticollis?
a. Yes
b. No
c. Not sure

4. How important is it for parents to have easy and free access to information about torticollis?

a. Not important

b. Somewhat unimportant

c. Neutral

d. Somewhat important

e. Important

5. Would you use a website designed to provide guidance on how to identify if your child could potentially have torticollis?
a. Yes
b. No
c. Not sure
6. Have you ever used a website that provides information on torticollis?

a. Yes: The website(s) has been very helpful b. Yes: The website(s) has been somewhat helpful

c. Yes: The website(s) has not been helpful

d. No

e. Not sure

7. How important is it that the website provides information on non-normal development pertaining to torticollis?

a. Not important

b. Somewhat unimportant

c. Neutral

d. Somewhat important

e. Important

8. How important is it that the website provides information on what to expect when the pediatrician/physical therapist assesses a child for torticollis?

a. Not important

b. Somewhat unimportant

c. Neutral

d. Somewhat important

e. Important

9. How important is it for the website to provide parents with information on what steps to take if their child is diagnosed with torticollis (e.g., attending physical therapy)?

a. Not important

b. Somewhat unimportant

c. Neutral

d. Somewhat important

e. Important

10. How important is it that the website uses photographs to demonstrate suggestions and activities?
a. Not important
b. Somewhat unimportant
c. Neutral
d. Somewhat important
e. Important

11. How important is it that the website provides information to parents on local programs and family resources such as systems of early intervention (e.g., Infants and Toddlers Program)?
a. Not important
b. Somewhat unimportant
c. Neutral 
d. Somewhat important

e. Important

12. Rank the following website features in order of importance; keep in mind that the website is for parents and is about torticollis. If not applicable, please click "N/A".

a. List of what to expect at assessment with the pediatrician

b. List of what to expect at assessment with the physical therapist

c. List of what to expect at appointments with pediatricians and physical therapist

d. List of exercises to do with a child

e. Resources list (e.g., systems of early intervention)

13. Please tell us a little bit about yourself, are you a mother, father, or other legal guardian of a young child?

a. Mother

b. Father

c. Grandmother who is the legal guardian of a young child

d. Grandfather who is the legal guardian of a young child

e. Other legal guardian of a young child

14. Are you male or female?

a. Male

b. Female

c. Prefer not to say

15. Please indicate your year of birth

16. What race/ethnicity best describes you?

a. American Indian or Alaskan Native

b. Asian

c. Black or African American

d. Hispanic or Latinx

e. Other (please specify):

17. What is your yearly total household income?

a. Less than $\$ 20,000$

b. $\$ 20,000$ to $\$ 34,999$

c. $\$ 35,000$ to $\$ 49,999$

d. $\$ 50,000$ to $\$ 74,999$

e. $\$ 75,000$ to $\$ 99,999$

f. $\$ 100,000$ to $\$ 149,999$

g. $\$ 150,000$ to $\$ 199,999$

h. $\$ 200,000$ or more

\section{Appendix B}

1. How much do you know about torticollis?

a. Nothing

b. A little

c. A lot

2. Have you worked with a child with torticollis before?

a. Yes

b. No

c. Not sure

Torticollis is a condition in which the sternocleidomastoid muscle (SCM), a muscle in the neck, is shortened. As a result, the head is tilted toward one side and rotated away from the shortened SCM. This muscle may have been shortened at birth (due to intrauterine crowding) or developed shortly after birth. Early treatment of torticollis is suggested and encouraged. Some signs of trauma on this muscle include bruising and swelling. Torticollis may lead to plagiocephaly (flattened skull), imbalance, hip dysplasia, vision and hearing problems, and scoliosis.

3. Do you think a website could help parents learn about torticollis?
a. Yes
b. No
c. Not sure

4. How important is it for parents to have easy and free access to information about torticollis?

a. Not important

b. Somewhat unimportant

c. Neutral

d. Somewhat important

e. Important

5. How important is it for you as a professional provider to inform all parents about torticollis?
a. Not important
b. Somewhat unimportant
c. Neutral
d. Somewhat important
e. Important

6. Do you currently have any resources available for parents of children with torticollis?

a. Yes: The resources we have are helpful for parents and does not need improvement

b. Yes: The resources we have are helpful for parents but 
needs improvement

c. Yes: The resources we have are not helpful for parents and improvement is needed

d. No

e. Not sure

7. How important is it that the website provides information on non-normal development pertaining to torticollis?

a. Not important

b. Somewhat unimportant

c. Neutral

d. Somewhat important

e. Important

8. How important is it that the website provides information on what to expect when the pediatrician/physical therapist assesses a child for torticollis?
a. Not important
b. Somewhat unimportant
c. Neutral
d. Somewhat important
e. Important

9. How important is it for the website to provide parents with information on what steps to take if their child is diagnosed with torticollis (e.g., attending physical therapy)?
a. Not important
b. Somewhat unimportant
c. Neutral
d. Somewhat important
e. Important

10. How important is it that the website uses photographs to demonstrate suggestions and activities?
a. Not important
b. Somewhat unimportant
c. Neutral
d. Somewhat important
e. Important

11. How important is it that the website provides information to parents on local programs and family resources such as systems of early intervention (e.g., Infants and Toddlers Program)?
a. Not important
b. Somewhat unimportant
c. Neutral

d. Somewhat important

e. Important

12. Rank the following website features in order of importance; keep in mind that the website is for parents and is about torticollis. If not applicable, please click "N/A".

a. List of what to expect at assessment with the pediatrician

b. List of what to expect at assessment with the physical therapist

c. List of what to expect at appointments with pediatricians and physical therapist

d. List of exercises to do with a child

e. Resources list (e.g., systems of early intervention)

13. If the website could provide more information to parents on certain organizations that can provide the child or family additional help, please rank the possible referral organizations in order of importance. If not applicable, please click "N/A".

a. Early Head Start/Head Start: educational program for children from families with low income

b. Early Intervention: a family centered system of services for young children with developmental delays and disabilitiesand their families

c. Home Visiting Program: supports pregnant women and families and helps parents of children from birth to age 5 tap the resources and develop the skills they need to raise children who are physically, socially and emotionally healthy and ready to learn

\section{d. Licensed Childcare Centers \\ e. Pediatrician \\ f. Preschool Program}

g. SSI: Supplemental Security Income designed to help families who have children with disabilities and have little or no income meet their basic needs for food, clothing, and shelter

h. WIC: a special supplemental nutritional program for low income, pregnant, breastfeeding, and non- breastfeeding postpartum women, and to infants and children up to age five who are found to be at nutritional risk.

14. What is your profession (check all that apply)?

a. Childcare provider

b. Nanny

c. Nurse

d. Occupational therapist

e. Physical therapist

f. Physician (e.g., pediatrician, developmental pediatrician, family practitioner)

g. Psychologist 
h. Service coordinator/case manager

i. Special education teacher

j. Speech and language pathologist

k. Teacher (e.g., preschool teacher, Early Head Start teacher

l. Other (please specify):

15. What race/ethnicity best describes you?

a. American Indian or Alaskan Native

b. Asian

c. Black or African American

d. Hispanic or Latinx

e. Other (please specify):

\section{References}

1. Vlimmeren VLA, Helders PJ, van Adrichem LN, Engelbert RH (2006) Torticollis and plagiocephaly in infancy: therapeutic strategies. Pediatr Rehabil 9(1): 40-46.

2. Per H, Canpolat M, Tümtürk A, Gumuş H, Gokoglu A, Yikilmaz A, et al. (2014) Different etiologies of acquired torticollis in childhood. Childs Nerv Syst 30(3): 431-440.

3. Young, MD, Young JL (2017) Conservative care of pediatric acquired torticollis: a report of 2 cases. J Chiropr Med 16(3): 252-256.

4. Flannery AB, Looman WS, Kemper K (2012) Evidence-based care of the child with deformational plagiocephaly, part II: management. J Pediatr Health Care 26(5): 320-331.

5. Wei JL, Schwartz KM, Weaver AL, Orvidas LJ (2001) Pseudotumor of infancy and congenital muscular torticollis: 170 cases. Laryngoscope 111(4 pt 1): 688-695.

6. Kuo AA, Tritasavit S, Graham JM (2014) Congenital muscular torticollis and positional plagiocephaly. Pediatr Rev 35(2): 79-87.

7. Stellwagen L, Hubbard E, Chambers C, Jones KL (2008) Torticollis, facial asymmetry and plagiocephaly in normal newborns. Archives of Disease in Childhood 93(10): 827-831.

8. Kaplan SL, Coulter C, Sargent B (2018) Physical therapy management of congenital muscular torticollis: a 2018 evidence-based clinical practice guideline from the apta academy of pediatric physical therapy. Pediatr Phys Ther 30(4): 240-290.

9. Davies MA, Terhorst L, Nakonechny AJ, Skukla N, El Saadawi G (2014) The development and effectiveness of a health information website designed to improve parents' self-efficacy in managing risk for obesity in preschoolers. Journal for Specialists in Pediatric Nursing 19(4): 316330 .

10. Marceau LD, Welch LC, Pemberton VL, Pearson GD (2016) Educating parents about pediatric research: children and clinical studies website qualitative evaluation. Qual Health Res 26(8): 1114-1122.

11. Albert D, Barracks SZ, Bruzelius E, Ward A (2014) Impact of a webbased intervention on maternal caries transmission and prevention knowledge, and oral health attitudes. Matern Child Health J 18(7): 17651771.

12. Nichter S (2016) A clinical algorithm for early identification and intervention of cervical muscular torticollis. Clin Pediatr (Phila) 55(6): 532-536.

13. Gutierrez D, Kaplan SL (2016) Aligning documentation with congenital muscular torticollis clinical practice guidelines: Administrative case report. Phys Ther 96(1): 111-120.

14. Whipple W (2014) Key principles of early intervention and effective practices: A crosswalk with statements from discipline specific literature. Regional research centre program, pp. 1-18.

15. Lillo-Navarro C, Medina-Mirapeix F, Escolar-Reina P, Montilla-Herrador J, Gomez-Arnaldos F, et al. (2015) Parents of children with physical disabilities perceive that characteristics of home exercise programs and physiotherapists' teaching styles influence adherence: A qualitative study. J Physiother 61(2): 81-86.

16.Ziviani J, Darlington Y, Feeney R, Rodger S, Watter P (2014) Early intervention services of children with physical disabilities: Complexity of child and family needs. Australian Occupational Therapy Journal 61(2): 67-75.

17.https://www.emedicinehealth.com/torticollis/article_em.htm\#what_ is_torticollis

18. https://kidshealth.org/en/parents/torticollis.html

19. https://www.healthline.com/health/torticollis

20.https: / /www.cedars-sinai.org/health-library/diseases-andconditions/t/torticollis.html

21. https://www.webmd.com/parenting/baby/what-is-torticollis\#1

22. Fletcher R, Vimpani G, Russell G, Keatinge D (2008) The evaluation of tailored and web-based information for new fathers. Child: Care. Health and Development 34(4): 439-446.

23. Hall CM, Bierman KL (2015) Technology-assisted interventions for parents of young children: Emerging practices, current research, and future directions. Early Childhood Research Quarterly 33: 21-32.

24. Kim W, Keefe RH (2010) Barriers to healthcare among Asian Americans. Soc Work Public Health 25(3): 286-295. 